\title{
Supported Employment - ein falsches Konzept für Deutschland - Pro
}

\author{
Supported Employment - A Wrong Policy for Germany? - Pro
}

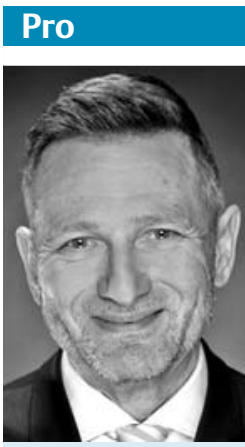

Heiko Kilian
Psychiatrische Rehabilitation und Arbeitsrehabilitation sind nach längerem Randdasein stärker ins Blickfeld geraten, seit sich die empirische Forschung der Thematik angenommen hat. Die Frage des optimalen Settings für Arbeitsrehabilitation bildete, wie beispielsweise in der Publikation von Bond, Drake und Becker [1], den Schwerpunkt der Untersuchungen. Hinsichtlich des Outcomes „Arbeitstätigkeit“ wurden die beiden Modelle „erst trainieren und dann platzieren“ (Prevocational Training, PVT), oft als „traditionelle Arbeitsrehabilitation“ bezeichnet, und „erst platzieren und dann trainieren“ (Supported Employment, SE) in verschiedenen internationalen Studien verglichen. Auf den ersten Blick ergeben sich überzeugende Signifikanzen zugunsten des SE, das seitdem mit Vehemenz als allein wirksames Instrument der Arbeitsrehabilitation propagiert wird.

Auf den zweiten Blick sind Zweifel angebracht. Die EQOLISE-Studie [2], die einzige Studie mit deutscher Beteiligung, leidet unter erheblichen methodischen Mängeln: 1. Verglichen wurde die langfristig angelegte SE mit einer RPK-Stichprobe und einer nur 6-wöchigen arbeitstherapeutischen Reha-Maßnahme, d.h., SE war allein durch die Dauer der Unterstützungsleistungen systematisch bevorzugt. 2. Folglich präferierten die Teilnehmer das SE-Angebot und hatten gegenüber den anderen beiden Settings negative Vorurteile, wie die Autoren selbst anführen.

Überraschenderweise waren dennoch die Unterschiede in Deutschland nicht signifikant, ein Ergebnis, über das in der aktuellen Diskussion erstaunlicherweise hinweg gesehen wird. Von den Verfechtern des SE-Ansatzes wird dies mit der Formulie- rung vernebelt, dass SE „im deutschen Sprachraum" nachweislich dem PVT überlegen sei, da in der Schweiz eine signifikante Überlegenheit des SE belegt ist. Jedoch stellt sich auch beim Berner Job Coach-Modell das Problem unterschiedlicher Dauer sowie nicht gleichwertiger finanzieller Bedingungen in den Vergleichsgruppen. Selbst Hoffmann [3] weist in diesem Zusammenhang darauf hin, dass finanzielle Arbeitgeberanreize zur Einstellung psychich beeinträchtigter Bewerber notwendig sind, um SE in Deutschland durchzusetzen.

Festzuhalten bleibt demnach: In Deutschland gibt es nach aktueller Studienlage hinsichtlich des Outcome-Kriteriums „Arbeitstätigkeit“" keine Unterschiede zwischen PVT und SE. Die Übertragbarkeit der internationalen Resultate auf Deutschland ist, wie auch Bond und Drake [4] ausführen, nicht nachgewiesen: „To summarize, the question of IPS transportability outside the US remains unanswered." Folglich erhielten beide Settings in den S3-Leitlinien „Psychosoziale Therapien bei schweren psychischen Störungen“ den gleichen Empfehlungsgrad B [5]. Warum ist aber SE sogar ein falsches Konzept für Deutschland?

Kein anderes Land auf der Welt verfügt über ein derartig gut ausgebautes Netz an Rehabilitationsmaßnahmen wie Deutschland $[6,7]$.

Im Bereich der beruflichen Rehabilitation gibt es eine umfassende und differenzierte Palette von kurzen Assessments über Vorbereitungs- und Trainingsmaßnahmen bis hin zu reinen Integrationsmaßnahmen, Aus- und Umschulungen sowie zahlreiche Programme von Jobcoaching und Supported Employment, letztere meist unter dem Begriff der „Unterstützten Beschäftigung (UB)“. Einrichtungen wie Berufliche Trainingszentren, RPK, Berufsförderungswerke, Berufsbildungswerke, Werkstätten und Integrationsfachdienste stellen - oft flächendeckend entsprechende Angebote ambulant wie stationär zur Verfügung. (Dass dabei Zu- gangshürden und Schnittstellenprobleme existieren, wird zu Recht kritisiert.)

Im Sozialgesetzbuch IX ist ein Rechtsanspruch auf entsprechende Leistungen festgeschrieben.

Nicht jeder psychisch beeinträchtigte Mensch kann und will sofort an einer Arbeitsstelle platziert werden, sondern viele bedürfen entsprechend ihrem Beeinträchtigungsgrad der Vorbereitung. Selbst Teilnehmer von SE-Programmen wie der UB absolvieren in vielen Fällen zuvor eine vorbereitende Trainingsmaßnahme. Entsprechend kommen bei den verschiedenen Leistungsanbietern auch Mischformen zwischen SE und PVT zunehmend zum Einsatz, wenn beispielsweise Berufliche Trainingszentren Nachbetreuungen nach Maßnahmeende regelhaft anbieten. Oft stoßen jedoch Weiterentwicklungen an bürokratische Hürden, wenn etwa die In-House-Phase einer von der Arbeitsagentur finanzierten Maßnahme mindestens $50 \%$ der Gesamtdauer umfassen muss, weil andernfalls das Vergaberecht eine Ausschreibung vorsieht.

Die Fachdienste und Rehabilitationsfachkräfte der Leistungsträger (Rentenversicherungen und Arbeitsagenturen, Jobcenter, Unfallversicherungen) stellen differenzierte Indikationen für die jeweils passenden Maßnahmen und bewilligen und finanzieren die entsprechenden Leistungen entsprechend dem jeweils individuellen Förderbedarf - „was passt wann für wen“.

Eine Propagierung des SE als einzig wirksame Maßnahme der beruflichen Wiedereingliederung würde einen Rückschritt für das deutsche Rehabilitationswesen bedeuten. Sie ignoriert die Versorgungsrealität, die in der Studienlage nicht erfasst wird.

Sie ignoriert auch die politischen, ökonomischen und sozialrechtlichen Unterschiede zwischen den angelsächsischen Ländern, der Schweiz und Deutschland. Ein vergleichsweise restriktives Arbeitsrecht mit hohem Schutz der Arbeitnehmerrechte hindert deutsche Arbeitgeber 
daran, Menschen mit Beeinträchtigung einzustellen.

Es besteht die Gefahr, dass hier das Kind mit dem Bade ausgeschüttet wird.

Somit bleibt als Fazit: Die Studienlage ergibt für Deutschland keine Überlegenheit des SE.

SE ist dennoch natürlich ein unverzichtbares Element in der rehabilitativen Versorgung, genauso unverzichtbar wie PVT, Ausbildungen, Umschulungen und Assessments.

Die Fragestellung der Studien mit ihrem holzschnittartigen Design SE versus PVT erscheint wenig nützlich und geht an der Versorgungsrealität in Deutschland vorbei. Es gilt vielmehr, diese differenziert und effektiv zu nutzen.

Offen bleibt auch, welches die wichtigsten Prädiktoren für eine erfolgreiche und dauerhafte Arbeitsintegration sind.

Für deutsche Verhältnisse verdienen beispielsweise folgende Fragen eine genauere Betrachtung:

- Welche Menschen welchen Beeinträchtigungsgrads profitieren von welchen Maßnahmen in welchem Ausmaß?

- Wie lassen sich Indikationen für bestimmte Einrichtungen, Maßnahmetypen oder Settings genauer beschreiben?

- Welche Elemente bestehender Maßnahmen sind stärker, welche weniger wirksam?

- Welche weiteren Variablen bestimmen den Erfolg arbeitsrehabilitativer Maß- nahmen? z. B. Welche Personen benötigen zeitlich unbefristete Unterstützung, für welche wäre sie nicht erforderlich oder sogar kontraindiziert?

Abgesehen von Deutschland legt die aktuelle Studienlage die Hypothese nahe, dass vor allem die Dauer der Unterstützung, und möglicherweise nicht primär das Setting, für die Nachhaltigkeit der Arbeitsintegration entscheidend ist.

Die für SE stets implizierte Forderung nach unbefristeter Unterstützung erscheint da folgerichtig, lässt jedoch außer Acht, dass dies für bestimmte Fälle und Diagnosegruppen auch kontraindiziert ist, weil es Regressionseffekte auslöst. Muss es aber nicht auch generell ein Ziel sein, möglichst viele Betroffene soweit zu verselbständigen, dass sie nicht auf dauerhafte Unterstützung angewiesen sind? Letztendlich kann es nur darum gehen, für jeden Betroffenen entsprechend seinem Beeinträchtigungsgrad, beruflichen Hintergrund und seiner psychosozialen Begleitumstände die jeweils passende Maßnahme im passenden Setting anzubieten, um Teilhabe sicherzustellen.

Interessenkonflikt: Der Autor ist Geschäftsführer eines beruflichen Trainingszentrums, das sowohl PVT-Maßnahmen als auch SE-Maßnahmen anbietet.

\section{Literatur \\ 1 Bond G, Drake R, Becker D. An update on ran- domized controlled trials of evidence based}

supported employment. Psychiatr Rehab J 2008; 4: 280-290

2 Burns T, Catty J, Becker T et al. The effectiveness of supported employment for people with severe mental illness: a randomised controlled trial. Lancet 2007; 370: $1146-$ 1152

3 Hoffmann H. Was macht Supported Employment so überlegen? Die Psychiatrie 2013; 10: $95-101$

4 Bond G, Drake R, Becker D. Generalizability of the Individual Placement and Support (IPS) model of supported employment outside the US. World Psychiatry 2012; 11: $32-39$

5 DGPPN, Hrsg. S3-Leitlinie Psychosoziale Therapien bei schweren psychischen $\mathrm{Er}$ krankungen. Berlin: Springer; 2012

6 Weisbrod M, Wirtz G, Fass $R$ et al. Realität und Zukunft der beruflichen Rehabilitation. Neurotransmitter 2015; 26: 30-37

7 Hammel Y, Kilian H, Schäfer F. Berufliche Rehabilitation psychisch Kranker - ein Überblick. Nervenheilkunde 2013; 6: 358-362

Sie haben eine eigene Meinung zu diesem

Thema? Dann schreiben Sie uns an: psychiat-praxis@thieme.de!

Korrespondenzadresse

Dipl.-Psych. Heiko Kilian c/o BTZ Rhein-Neckar

Lempenseite 46

69168 Wiesloch

Heiko.Kilian@btz.srh.de

Bibliografie

Dol http://dx.doi.org/

10.1055/s-0042-109424

Psychiat Prax 2016; 43: 242-243

(c) Georg Thieme Verlag KG

Stuttgart · New York

ISSN 0303-4259 